\title{
PREVALÊNCIA E FATORES ASSOCIADOS À PRÁTICA DA AMAMENTAÇÃO DE CRIANÇAS QUE FREQÜENTAM UMA CRECHE COMUNITÁRIA
}

\author{
PREVALENCE AND FACTORS ASSOCIATED WITH BREASTFEEDING \\ FOR CHILDREN ATTENDING A PUBLIC COMMUNITARIANCHILD \\ DAYCARE CENTER
}

\section{PREVALENCIA Y FACTORES ASOCIADOS A LA PRÁCTICA DE LA LACTANCIA MATERNA DE NIÑOS QUE ASISTEN A UNA GUARDERÍA COMUNITARIA}

\author{
Maria Helena do Nascimento Souza* \\ Vitória Regina Domingues Sodré ${ }^{* *}$ \\ Fabíola Nogueira Ferreira da Silva ${ }^{* * *}$
}

\begin{abstract}
RESUMO
É inquestionável o valor do leite materno como fonte nutricional exclusiva para a criança nos primeiros seis meses de vida, onde dentre vários benefícios evidencia-se a prevenção da morbi-mortalidade infantil. Este estudo teve como objetivos: avaliar a prevalência do aleitamento materno e analisar os fatores associados à prática da amamentação em uma Creche Comunitária. Método: A abordagem metodológica foi de natureza quantitativa, sendo o estudo do tipo seccional realizado com 46 mães de crianças menores de dois anos. Os dados foram coletados no período de junho a julho de 2013 e analisados com o auxílio do programa SPSS versão 19 (Statistical Package for the Social Sciences). A associação entre as variáveis foi investigada com os testes: quiquadrado, exato de Fischer, razão de prevalência e considerou-se o intervalo de confiança de $95 \%$. Os resultados mostraram que a prevalência do aleitamento materno exclusivo (AME) foi de 34,8\% e de aleitamento materno (AM) foi de $57,1 \%$. Os principais motivos referidos pelas mães para o desmame precoce foram: necessidade de trabalhar $(26,1 \%)$, orientações de familiares ou profissionais de saúde (19,6\%), problemas de saúde materna $(19,6 \%)$ e recusa da criança $(19,6 \%)$. Não foram observadas associações entre os fatores determinantes da prática da amamentação das crianças da creche estudada. Conclui-se que as prevalências de AME e de AM apresentaram-se abaixo do esperado pela Organização Mundial de Saúde e que o conhecimento dos fatores associados à amamentação pode constituir em um importante subsídio para a implementação de ações voltadas para a promoção, proteção e apoio ao aleitamento materno.
\end{abstract}

Palavras chave: Aleitamento materno, saúde da criança, creches, saúde pública.

\footnotetext{
* Enfermera. Professora do Departamento de Enfermagem de Saúde Pública da Escola de Enfermagem Anna Nery da Universidade Federal do Rio de Janeiro (UFRJ).Email: mhnsouza@yahoo.com.br

** Acadêmica do $8^{\circ}$ período do Curso de Graduação em Enfermagem da EEAN/UFRJ. Email: vitoria.regina1@hotmail.com

*** Acadêmica do 7º período do Curso de Graduação em Enfermagem da EEAN/UFRJ. Email: fabiola_bibila@hotmail.com
} 


\begin{abstract}
Breast milk's worth as exclusive nutritional source for children in the first six months of life is unquestionable, and, among its many benefits, the prevention of child morbimortality is evidenced. This study aims to evaluate the prevalence of breastfeeding and analyze the factors associated with breastfeeding at a Public Communitarian Daycare Center. The theoretical approach was quantitative, being the cross-sectional study performed in a sample of 46 mothers of children aging less than two years old. Data were collected between June and July 2013 and analyzed by using the software SPSS, version 19, (Statistical Package for the Social Sciences). For the association between variables, specific tests were used: Chi-Squared Test, Fischer's exact test and prevalence ratio. In addition, the considered confidence interval was $95 \%$. The results have shown that the exclusive breastfeeding prevalence rate (AME) was $34.8 \%$ and the breastfeeding rate (AM) was $57.1 \%$. The main reasons referred by the mothers for premature weaning were: work necessity $(26.1 \%)$, guidance from family members or health professionals (19.6\%), maternal health problems (19.6\%) and child's refusal (19.6\%). Associations between relevant factors of breastfeeding for children attending the daycare center studied were not observed. The study has concluded that the prevalence of AME and AM were lower than the expected by the World Health Organization, and knowledge about the factors associated with breastfeeding can constitute an important subside for the implementation of action focusing on promotion, protection and support of maternal breastfeeding.
\end{abstract}

Key words: Breast feeding, child health, child day care centers, public health.

\title{
RESUMEN
}

Es incuestionable el valor de la leche materna como fuente nutricional exclusiva para el niño en los primeros seis meses de vida, donde se evidencia, entre varios beneficios, la prevención de la morbimortalidad infantil. Este estudio tuvo como objetivos: evaluar la prevalencia de la lactancia materna y analizar los factores asociados a esta práctica en una Guardería Comunitaria. El enfoque metodológico fue de naturaleza cuantitativa del tipo seccional. Los datos fueron recolectados con 46 madres de niños menores de dos años, en el período de junio a julio de 2013 y analizados con la ayuda del programa SPSS, versión 19 (Statistical Package for the Social Sciences). La asociación entre las variables se investigó con los tests: chi cuadrado, exacto de Fisher, razón de prevalencia y se consideró el intervalo de confianza del 95\%. Los resultados mostraron que la prevalencia de la lactancia materna exclusiva (LME) fue del 34,8\% y de lactancia materna (LM) fue del 57,1\%. Los principales motivos relatados por las madres para el destete precoz fueron: necesidad de trabajar (26,1\%), orientaciones de familiares o profesionales de salud (19,6\%), problemas de salud materna $(19,6 \%)$ y rechazo del niño $(19,6 \%)$. No se observaron asociaciones entre los factores determinantes de la práctica del amamantamiento de los niños. Se concluyó que la prevalencia de lactancia materna se presentó por debajo de lo esperado por la Organización Mundial de Salud y que el conocimiento que tienen las madres sobre qué factores se asocian a una baja prevalencia de LME o LM no exclusiva puede constituir una importante contribución para la implementación de acciones de promoción, protección y apoyo a la lactancia materna.

Palabras clave: Lactancia materna, salud infantil, jardines infantiles, salud pública.

Fecha recepción: 03/03/14 Fecha aceptación: 23/03/15 


\section{INTRODUÇÃO}

O aleitamento materno proporciona inúmeros benefícios para o crescimento e desenvolvimento da criança e constitui uma importante medida para a prevenção da morbi-mortalidade infantil. É inquestionável o valor do leite materno do ponto de vista nutricional, anti-infeccioso, imunológico, econômico, anticoncepcional, emocional, entre outros; sendo de consenso que este leite deve ser utilizado como fonte exclusiva de nutrientes para a criança até os seis meses de idade (1-4).

As estratégias de promoção, proteção e apoio da amamentação, têm se transformado em linhas prioritárias de interesse para os formuladores de políticas públicas, sendo enfatizadas no modelo assistencial dos programas e estratégias do Sistema Único de Saúde tais como: Programa de Atenção Integral à Saúde da Mulher, Programa de Atenção Integral à Saúde da Criança, Atenção Integrada às Doenças Prevalentes na Infância, Política Nacional de Alimentação e Nutrição (PNAN), Estratégia Saúde da Família, entre outros $(4,5)$.

Apesar dos esforços dos órgãos governamentais e não governamentais para que sejam implementadas ações que visem promover a prática do aleitamento materno exclusivo (AME), apoiar a mulher durante a fase da amamentação e aumentar as taxas de duração do aleitamento materno (AM), diversos estudos mostram que ainda há um alto índice de desmame precoce, ou seja, de mulheres que não amamentam exclusivamente seus filhos até o sexto mês de idade. E conseqüentemente há uma baixa prevalência de mulheres que mesmo após a introdução de outros alimentos, mantém o leite materno como uma das fontes nutritivas para a criança até 24 meses de vida, conforme preconizado pela Organização Mundial de Saúde (1, 6-8).

Segundo dados da II Pesquisa Nacional de Aleitamento Materno, realizada nas capi- tais e Distrito Federal em 2008, a prevalência nacional de aleitamento materno exclusivo foi de $41 \%$, sendo que na região Sudeste esta taxa foi abaixo da média nacional $(39,4 \%)$, demonstrando assim que no Brasil a prevalência de amamentação exclusiva está aquém do recomendado pelo Ministério da Saúde e Organização Mundial da Saúde (9).

Sabe-se que um dos motivos da não manutenção do aleitamento materno é devido à crescente necessidade da mulher estar inserida no mercado de trabalho e poder contribuir para o aumento do rendimento familiar. Frente a realidade, o ambiente das creches, escolas ou centros educacionais, tem-se constituído o local onde as crianças, desde os primeiros meses de vida permanecem grande parte do dia, durante o tempo em que a mãe ou responsável exerce sua atividade laboral (10-12).

No contexto das creches, nota-se que as crianças menores de dois anos tem maior possibilidade de contraírem infecções e outros agravos característicos desta faixa etária, quando comparadas com aquelas que são cuidadas exclusivamente em casa. Pois, nestes ambientes coletivos, elas convivem diariamente e de forma prolongada tanto com crianças sadias quanto com aquelas que apresentam alguma morbidade $(10,12,13)$.

Nesta perspectiva, ressalta-se a importância do desenvolvimento de medidas voltadas para a promoção da saúde, prevenção de agravos mais comuns na infância entre as crianças que freqüentam creches, de modo que tais instituições se tornem um ambiente mais saudável e seguro $(7,12)$. Dentre as ações de promoção da saúde no primeiro ano de vida, destaca-se o incentivo do aleitamento materno exclusivo até o sexto mês ou até o quarto mês, no caso das mães que trabalham e que gozam da licença à maternidade.

O conhecimento da prevalência da amamentação de crianças que freqüentam creches, bem como dos fatores associados a esta prática, poderá constituir em um subsídio para a atuação de profissionais de saúde, na 
implementação de medidas eficazes voltadas para a promoção do aleitamento materno e diminuição da morbi-mortalidade infantil (5).

Diante do exposto, os objetivos deste estudo foram: avaliar a prevalência do aleitamento materno e identificar os fatores associados à prática da amamentação de crianças que freqüentam uma creche comunitária.

\section{MATERIAL E MÉTODO}

O presente estudo foi do tipo seccional analítico, de abordagem quantitativa e foi desenvolvido em uma creche comunitária situada na zona sul do Município do Rio de Janeiro. A escolha da referida creche para a realização desta pesquisa deu-se por se tratar de uma instituição que atende crianças de classe baixa que vivem em condições socioeconômicas e sanitárias precárias, consideradas de risco para a saúde infantil. E pelo fato de que a comunidade estudada constituir em um campo de prática de alunos do curso de graduação em enfermagem da Universidade Federal do Rio de Janeiro.

A população do estudo foi constituída por 46 mães de crianças menores de vinte e quatro meses que estavam freqüentando a referida creche, no período de junho e julho de 2013, ocasião da coleta dos dados. Todas as mães foram convidadas a participar da pesquisa e após terem recebido esclarecimento sobre os objetivos do estudo, concordaram e assinaram o Termo de Consentimento Livre e Esclarecido.

Os dados foram coletados mediante uma entrevista às mães, com base em formulário contendo questões referentes: às condições sócio-econômicas, características relacionadas à gestação, parto, amamentação e saúde da criança; que foram consideradas variáveis de exposição.

O tempo de aleitamento materno exclusivo (AME), ou seja, o tempo em que a criança recebeu apenas o leite materno, sem adição de outros líquidos ou alimentos sólidos, com exceção de vitaminas, sais de reidratação oral ou medicamentos; foi considerado a variável de desfecho. Considerou-se ainda, o tempo de aleitamento materno (AM) definido como o número de meses em que a criança recebeu leite materno (direto da mama ou ordenhado), independentemente de receber ou não outros alimentos (2).

A classificação do estado nutricional das crianças deu-se por meio da aferição do peso e estatura das mesmas e cálculo do escore $\mathrm{Z}$ de peso para estatura (P/E). Foram consideradas obesas e sobrepeso as crianças que apresentaram o escore $\mathrm{P} / \mathrm{E}$ igual ou superior a 2.

Este estudo recebeu aprovação do Comitê de Ética e Pesquisa da Escola de Enfermagem Anna Nery da Universidade Federal do Rio de Janeiro em 03/04/2013 sob o parecer no 236.478 registrado, na Plataforma Brasil. E a pesquisa foi autorizada pela direção da referida creche.

Para a formação do banco de dados utilizou-se o Software Epi-info versão 3.5.2, para os cálculos do estado nutricional foi utilizado o programa AnthroPlus (14) e para a análise estatística das variáveis utilizou-se o programa SPSS versão 19 (Statistical Package for the Social Sciences). A associação entre as variáveis de exposição e desfecho foi investigada com os testes: qui-quadrado, exato de Fischer, razão de prevalência, considerando o intervalo de confiança de $95 \%$ e o nível de significância menor que 0,05 .

\section{RESULTADOS}

Os resultados mostraram que das 46 mães de crianças menores de 2 anos matriculadas na creche estudada,16 mães haviam amamentado exclusivamente até o sexto mês, o que corresponde a uma prevalência de AME igual a 34,8\%. Já a prevalência de aleitamen- 
to materno (AM) após o sexto mês de vida da criança foi de $74,1 \%$, revelando que após o desmame precoce algumas mães continuaram oferecendo seu leite para a criança, juntamente com outros alimentos (Gráfico 1).

Em relação às características das mães verificou-se que: $89,1 \%$ tinham mais de 20 anos de idade, $58,7 \%$ possuíam até o ensino fundamental, $67,4 \%$ viviam com o companheiro, $65,2 \%$ trabalharam durante a gestação, sendo que destas $83,3 \%$ tiveram licença à maternidade, 52,2\% eram multíparas, $73,3 \%$ não participaram de grupos de gestantes, 52,2\% realizaram parto cesárea e 63\% contaram com apoio da rede social durante a fase da amamentação (Tabela 1).

Gráfico 1. Prevalência de aleitamento materno exclusivo (AME) e de aleitamento materno (AM) em crianças menores de dois anos de uma creche comunitária, Rio de Janeiro, 2013 ( $\mathrm{n}=46)$.

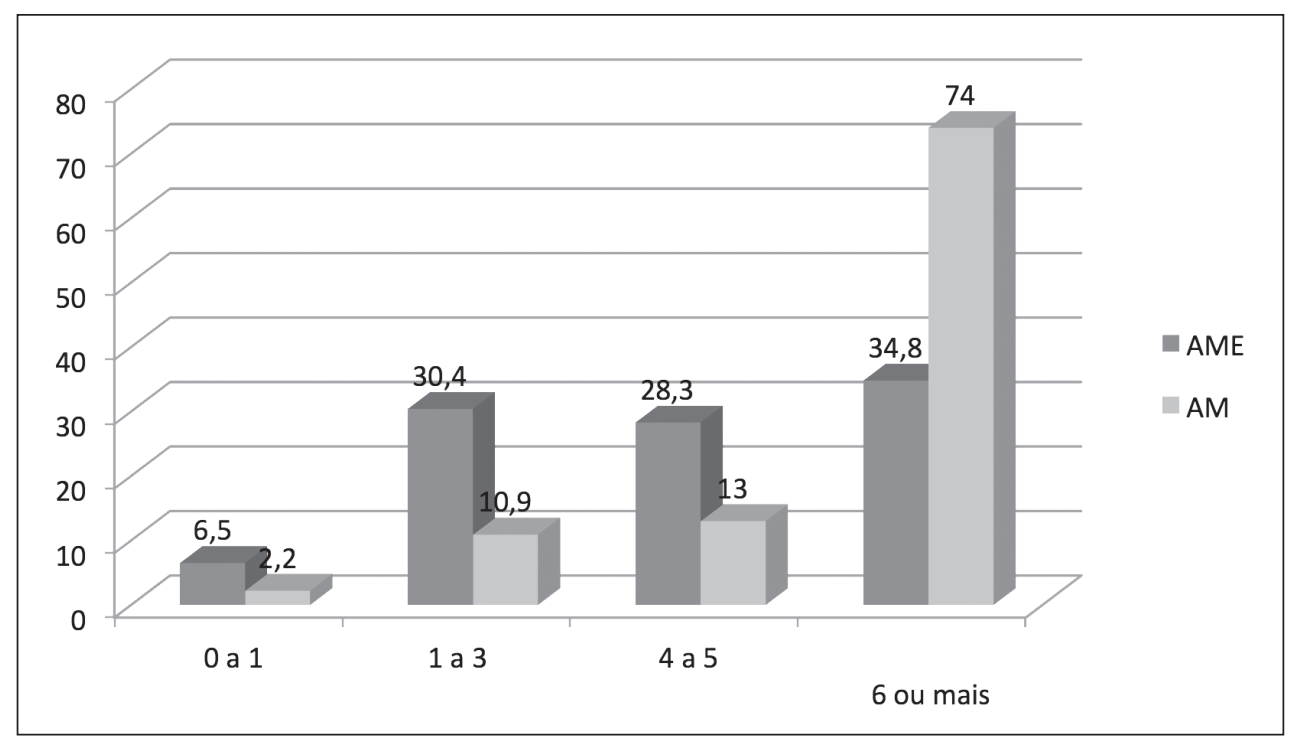

Tabela 1. Características maternas associadas ao aleitamento materno exclusivo em crianças menores de 2 anos que freqüentam uma creche comunitária do município do Rio de Janeiro, $2013(n=46)$.

\begin{tabular}{lccccc}
\hline Características maternas & $\begin{array}{c}\text { Total } \\
\mathbf{n} \%\end{array}$ & $\begin{array}{c}\text { AME } \\
\mathbf{n} \%\end{array}$ & RP & IC 95\% & p \\
\hline $\begin{array}{l}\text { Idade (em anos) } \\
<20\end{array}$ & $05(10,9)$ & $0(0,0)$ & 0 & $0,0-1,5$ & $0,14^{\varepsilon}$ \\
$>20$ & $41(89,1)$ & $16(100,0)$ & 1 & & \\
Escolaridade & & & & & \\
Ensino Médio ou Superior & $19(41,3)$ & $09(56,3)$ & 1,82 & $0,7-4,5$ & $0,13^{\&}$ \\
Até o Ensino Fundamental & $27(58,7)$ & $07(43,7)$ & 1 & & \\
Situação Conjugal & & & & & \\
Casada ou vive em união & $31(67,4)$ & $10(62,5)$ & 0,8 & $0,3-2,2$ & $0,60^{\&}$ \\
Solteira & $15(32,6)$ & $6(37,5)$ & 1 & & \\
\hline
\end{tabular}


Continuação Tabela 1.

\begin{tabular}{lccccc}
\hline Trabalhou na gestação & & & & & \\
Sim & $30(65,2)$ & $12(75,0)$ & 0,8 & $0,5-1,2$ & $0,24^{\varepsilon}$ \\
Não & $16(34,8)$ & $04(25,0)$ & 1 & & \\
Licença a maternidade & & & & & \\
Sim & $25(83,3)$ & $10(83,3)$ & 1 & $0,3-1,0$ & $0,68^{\varepsilon}$ \\
Não & $05(16,7)$ & $02(16,7)$ & 1 & & \\
Paridade & & & & & \\
Multípara & $24(52,2)$ & $06(37,5)$ & 1,37 & $0,9-2,1$ & $0,14^{\&}$ \\
Primípara & $22(47,8)$ & $10(62,5)$ & 1 & & \\
Participação em grupo de gestantes & & & & & \\
Sim & $12(26,7)$ & $4(26,7)$ & 1 & $0,3-2,5$ & $0,64^{\varepsilon}$ \\
Não & $33(73,3)$ & $11(73,3)$ & 1 & & \\
Tipo de parto & & & & & \\
Normal & $22(47,8)$ & $06(37,5)$ & 0,65 & $0,2-1,6$ & $0,31^{\&}$ \\
Cesárea & $24(52,2)$ & $10(62,5)$ & & & \\
Recebeu apoio da rede social na fase da amamentação & & & & & \\
Sim & $29(63,0)$ & $10(63,0)$ & 0,97 & $0,40-2,67$ & $0,95^{\&}$ \\
Não & $17(37,0)$ & $06(37,0)$ & & & \\
\hline
\end{tabular}

\& valor de $\mathrm{p}$ do teste de qui-quadrado de Pearson, ${ }^{\mathfrak{E}}$ valor de $\mathrm{p}$ do teste Exato de Fisher RP = Razão de Prevalência, IC 95\%= Intervalo de Confiança de 95\%

$\mathrm{AME}=$ Aleitamento materno exclusivo

Ao analisar a associação entre as características das mães de crianças menores de 2 anos que freqüentam a creche estudada e a prática da amamentação exclusiva até o sexto mês pós-parto, observou-se que não houve associação significativa entre o AME e tais variáveis maternas. No entanto, verificou-se que as mães que tiveram maior chance de amamentar foram aquelas que: possuíam idade superior a 20 anos, ensino médio ou superior, eram casadas, que trabalharam na gestação e tiveram licença à maternidade, eram primíparas, tiveram parto cesárea e receberam apoio de membros da rede social durante à amamentação (Tabela 1).

Os principais motivos referidos pelas mães para o desmame precoce foram: necessidade de trabalhar $(26,1 \%)$, orientações de familiares ou profissionais de saúde $(19,6 \%)$, problemas de saúde materna $(19,6 \%)$, recusa da criança $(19,6 \%)$ e "leite fraco" (15\%) (Gráfico 2).
Em relação ás características das crianças estudadas: $82,6 \%$ encontravam-se na faixa etária de 1 a 2 anos de idade, 56,5\% eram do sexo feminino, $91,1 \%$ nasceram com peso superior a 2500 gramas, 58,7\% não recebeu leite materno na primeira hora de vida, $50,0 \%$ fizeram uso de chupeta, $89,1 \%$ nunca foram internadas e $12,5 \%$ foram classificadas como sobrepeso ou obesidade (Tabela 2).

Quanto aos fatores associados ao AME, não foi encontrada associação significativa entre o tempo de AME destas crianças e as demais variáveis da Tabela 2. No entanto, a prevalência do AME foi mais elevada entre as crianças: na faixa etária de 1 a 2 anos, do sexo feminino, com peso ao nascer acima de 2500 gramas, que foram amamentadas na primeira hora de vida, nunca ficaram internadas e entre aquelas cujo o estado nutricional foram classificado como normal. 
Gráfico 2. Motivos do desmame precoce em crianças menores de dois anos de uma creche comunitária, Rio de Janeiro, $2013(\mathrm{n}=46)$.

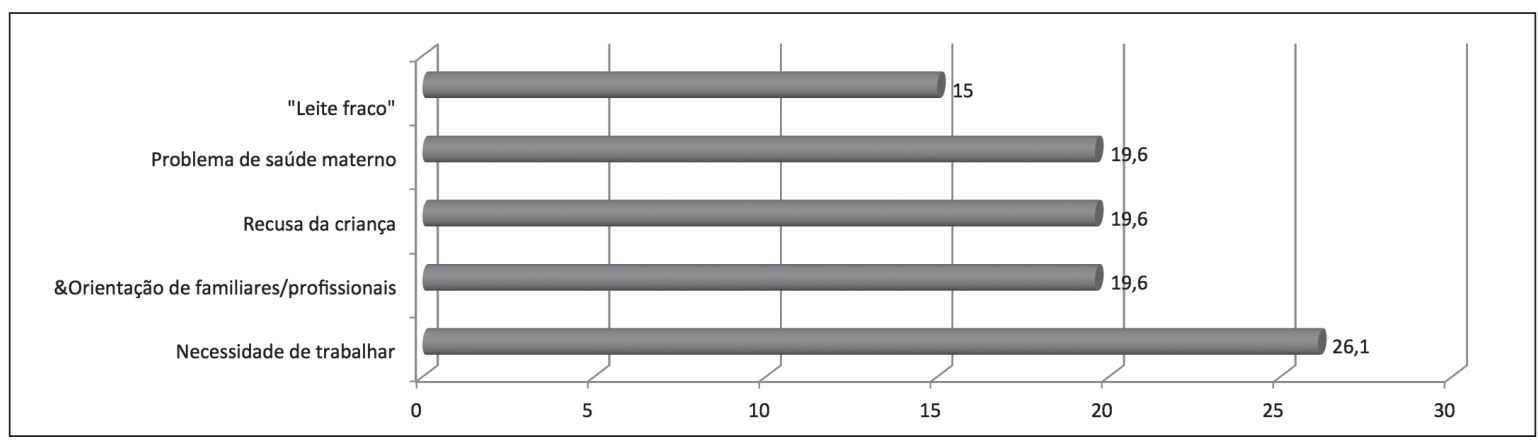

Tabela 2. Características das crianças menores de 2 anos que freqüentam uma creche comunitária associadas ao aleitamento materno exclusivo, município do Rio de Janeiro, 2013 $(\mathrm{n}=46)$.

\begin{tabular}{|c|c|c|c|c|c|}
\hline \multirow[t]{2}{*}{ Características das crianças } & Total & AME & \multirow[t]{2}{*}{$\mathbf{R P}$} & \multirow[t]{2}{*}{ IC 95\% } & \multirow[t]{2}{*}{$\mathrm{p}$} \\
\hline & n $\%$ & n $\%$ & & & \\
\hline \multicolumn{6}{|l|}{ Idade (em anos) } \\
\hline 1 a 2 & $38(82,6)$ & $14(87,5)$ & 1,47 & $0,5-9,0$ & $0.69^{2}$ \\
\hline$<1$ & $08(17,4)$ & $02(12,5)$ & 1 & & \\
\hline \multicolumn{6}{|l|}{ Sexo } \\
\hline Masculino & $20(43,5)$ & $05(31,0)$ & 0,59 & $0,2-1,5$ & $0.22^{8}$ \\
\hline Feminino & $26(56,5)$ & $11(69,0)$ & 1 & & \\
\hline \multicolumn{6}{|l|}{ Peso as nascer } \\
\hline$>2500$ & $41(91,1)$ & $15(93,7)$ & 1,46 & $0,4-29$ & $0.55^{5}$ \\
\hline$<2500$ & $04(8,9)$ & $01(6,3)$ & 1 & & \\
\hline \multicolumn{6}{|l|}{ Mamou na primeira hora } \\
\hline Sim & $19(41,3)$ & $09(56,3)$ & 1,82 & $0,7-4,5$ & $0.13^{8}$ \\
\hline Não & $27(58,7)$ & $07(43,7)$ & 1 & & \\
\hline \multicolumn{6}{|l|}{ Uso de chupeta } \\
\hline Sim & $23(50,0)$ & $08(50,0)$ & 1 & $0,4-2,5$ & $1^{\&}$ \\
\hline Não & $23(50,0)$ & $08(50,0)$ & 1 & & \\
\hline \multicolumn{6}{|l|}{ Já ficou internado } \\
\hline Não & $41(89,1)$ & $14(87,5)$ & 0,81 & $0,3-4,9$ & $0.55^{2}$ \\
\hline Sim & $05(10,9)$ & $02(12,5)$ & 1 & & \\
\hline \multicolumn{6}{|l|}{ Estado nutricional } \\
\hline Normal & $40(87,0)$ & $14(87,5)$ & 1,05 & $0,3-3,5$ & $0.65^{2}$ \\
\hline Sobrepeso/obesidade & $06(13,0)$ & $02(12,5)$ & 1 & & \\
\hline
\end{tabular}

${ }^{8}$ valor de p do teste de qui-quadrado de Pearson, ${ }^{\text {E}}$ valor de p do teste Exato de Fisher RP = Razão de Prevalência, IC95\%= Intervalo de Confiança de 95\%

$\mathrm{AME}=$ Aleitamento materno exclusivo 


\section{DISCUSSÃO E CONCLUSÃO}

A baixa prevalência de aleitamento materno exclusivo, encontrada neste estudo, vem ao encontro dos resultados obtidos em outras pesquisas que também evidenciaram taxas abaixo do preconizado pela Organização Mundial da Saúde. Estudo realizado em uma Unidade de Saúde da Família do Rio Grande do Sul revelou que a prevalência de AME de crianças atendidas foi de 33,7\% (15). E uma investigação realizada no Paraná também mostrou uma baixa prevalência de AME, equivalente a $36,8 \%$ (16).

A Organização Mundial da Saúde (OMS) e o Ministério da Saúde recomendam o aleitamento materno exclusivo por seis meses e que após este período o leite materno seja complementado até os dois anos, pois a prática do desmame precoce pode causar prejuízos à saúde da criança. Entre esses prejuízos estão o aumento de episódios de diarréia; maior número de hospitalizações por doença respiratória; risco de desnutrição se os alimentos introduzidos forem nutricionalmente inferiores; menor absorção de nutrientes importantes do leite materno, como o ferro e o zinco; menor eficácia da lactação como método anticoncepcional; menor duração do aleitamento materno $(5,6)$. No entanto, a baixa prevalência de AME encontrada no presente estudo pode ser justificada pelo fato das mães optarem pelo desmame precoce frente a necessidade de retornarem as atividades laborais e a preocupação com a adaptação da criança à alimentação oferecida na creche. Apesar da creche estudada, matricular crianças a partir do quarto mês de vida, algumas mães referiram ter oferecido para a criança alimentos diferente do leite materno até mesmo antes do quarto mês de vida, período em que teriam direito à licença à maternidade e teriam a possibilidade de amamentar exclusivamente a criança no domicílio.

Estudo realizado no cenário de creches no município de São Paulo revelou que apesar da duração média do AME ter sido de 2,9 meses (17), esta taxa foi superior a prevalência média de 1,8 meses, encontrada em 2006 nas capitais brasileiras (9). Para estes autores este aumento na duração da amamentação, observado entre as crianças das creches estudadas pode ter sido decorrente das iniciativas de proteção ao aleitamento materno instituídas no país nas últimas décadas e da possibilidade da creche ser um local de educação das mães ao informar os benefícios da amamentação (17).

Com relação à idade materna, os resultados deste estudo corroboram com os achados de um estudo realizado no contexto de uma comunidade de Goiânia-GO com 30 mães de crianças menores de um ano, onde se verificou que a faixa etária predominante foi de 26 a 30 anos (43,3\%) (18).

A baixa escolaridade materna e a necessidade de trabalhar fora de casa, observadas neste estudo, pode ser reflexo da situação de moradia desta população. Pois, a creche estudada está situada em uma comunidade em que as condições sócio-econômicas, familiares, de escolaridade, trabalho, saúde, habitação, bem como a presença de situações de violência, são evidenciadas claramente, refletindo as condições de pobreza e as precárias condições de vida da maioria dos moradores desta localidade (19).

O estado civil predominante foi casada/ vive em união $(67,4 \%)$. O fato da maioria das mulheres conviverem com seus companheiros/maridos pode influenciar positivamente no período de aleitamento materno, principalmente quando estas se encontravam nos primeiros meses pós-parto, gozando ou não da licença à maternidade. Durante a fase da amamentação, os companheiros juntamente com outros familiares ou membros da rede social, podem fornecer o suporte necessário à nutriz, quer seja pela presença constante, auxílio financeiro, ajuda na realização de tarefas domésticas, cuidados com a criança, ou 
apoio à mulher durante o período do aleitamento (20-22).

Com relação ao apoio à mulher que amamenta faz-se cada vez mais necessário o incentivo de atividades educativas em forma de grupos. Os dados obtidos desse estudo mostraram que um grande percentual das mães $(73,3 \%)$ não participaram de nenhum grupo de apoio à amamentação durante a gestação.

Para o sucesso do aleitamento materno é primordial que o incentivo e a promoção desta prática iniciem no período gestacional, durante o pré-natal, onde a gestante tem oportunidade de receber orientações individuais e em grupo sobre as mamas, o leite materno e a prática da amamentação, assim como discutir as vantagens, questões polêmicas e mitos relacionados ao aleitamento $(5,8$, 22). As atividades em grupo possibilitam que o profissional de saúde identifique e compreenda o processo do aleitamento materno em contextos socioculturais e familiares, e a partir dessa compreensão, possa cuidar tanto do binômio mãe-bebê e orientar a família. Diante desta perspectiva, os grupos de apoio comunitários podem constituir uma ferramenta valiosa para as equipes de saúde e serem eficientes para fornecer uma ajuda diária às mães que amamentam (21).

Quanto ao tipo de parto, verificou-se uma alta prevalência $(52,2 \%)$, não condizendo com o que é preconizado pela OMS, que as taxas de cesárea mantenham-se entre 5 e 15\% do número de partos totais (23). Há estudos que apontam o parto cesáreo como um fator de risco para a amamentação ao nascimento $(24,25)$, enquanto que outros não apresentaram nenhuma associação entre o parto cesárea e a duração da amamentação exclusiva $(26,27)$.

Os motivos para a interrupção da amamentação referidos pelas mães estudadas corroboram com aqueles apresentados em outros estudos que também apresentam as seguintes causas para o desmame precoce: leite fraco ou insuficiente, criança não quis mamar, problemas maternos, trabalho materno, recomendação médica, mitos populares, entre outros $(3,7,8)$.

Tais motivos são ainda presentes não apenas no contexto das comunidades, mas em entre mulheres de classes sociais mais elevadas, demonstrando a necessidade dos profissionais de saúde compreenderem as expectativas maternas em relação à amamentação tendo em vista o desempenho das funções domésticas, profissionais e sociais, a fim de contribuir para a ampliação dos conhecimentos quanto aos benefícios do leite materno para a saúde da criança, prevenção de doenças e promoção do crescimento e desenvolvimento saudável $(3,11,15,16)$.

Nota-se que o desmame precoce ocorre diante de períodos críticos onde surgem dificuldades, como fissuras, ingurgitamento, dor à sucção, angústia, ansiedade e outras $(7,17)$ ou diante da necessidade da mulher retornar ao trabalho. Nesse caso, quando há a possibilidade de deixar a criança em uma creche, muitas vezes, a mãe inicia precocemente a introdução de outros alimentos diferentes do leite materno, a fim de sentir-se segura de que a criança terá uma boa aceitação da dieta e conseqüentemente uma boa adaptação na creche, local em que a criança permanecerá grande parte do tempo, enquanto a mãe estiver trabalhando fora de casa.

Observou-se que um percentual $8,9 \%$ das crianças nasceu com baixo peso. Este valor foi superior a taxa de $6,1 \%$ encontrada na Pesquisa Nacional de Demografia e Saúde da Criança e da Mulher em 2006 (28). Sabe-se que o aumento do baixo peso ao nascer pode estar relacionado à prematuridade, menor poder aquisitivo da população, dificuldade no acesso e pouca disponibilidade de recursos de saúde, o que resulta em uma maior probabilidade de riscos à saúde e de incidência de mortalidade infantil $(15,18,26)$.

Todas as crianças do estudo foram amamentadas, o que é satisfatório, uma vez que o aleitamento materno é uma estratégia natu- 
ral de vínculo, afeto, proteção e nutrição para a criança e constitui a mais sensível, econômica e eficaz intervenção para redução da morbimortalidade infantil, além de ser um processo que envolve interação profunda entre mãe e filho, com repercussões no estado nutricional da criança, em sua habilidade de se defender de infecções, em sua fisiologia e no seu desenvolvimento cognitivo e emocional, além de ter implicações na saúde física e psíquica da mãe (2).

Do total de crianças, apenas 58,7\% foram amamentadas na primeira hora pós parto, o que contradiz a Organização Mundial da Saúde que preconiza a amamentação na primeira meia hora de vida como sendo uma das estratégias prioritárias para a promoção, proteção e apoio ao aleitamento materno, possibilitando o vínculo mãe-bebê, sendo uma prática que pode reduzir em $22 \%$ a mortalidade neonatal causadas por infecções (25).

O uso de chupeta foi identificado em metade da população estudada. De acordo com a Organização Mundial da Saúde (OMS) e o Fundo das Nações Unidas para a Infância (UNICEF) o não uso de chupeta e mamadeiras, constitui um dos fatores que previnem o desmame precoce. Contudo, neste estudo não foi verificado associação do AME e uso de chupeta. Segundo alguns autores é possível que o ato de sugar a chupeta iniba a amamentação, mas também é possível que as mães que têm problemas para amamentar utilizem as chupetas para acalmar seus bebês $(24,25)$. Além de acalmar o bebê, essa prática poderia também acalmar a própria mãe que, ansiosa ao ver o bebê chorando, utiliza essa prática para silenciá-lo.

Crianças desmamadas precocemente apresentam maior índice de morbidades e internação hospitalar, principalmente aquelas por infecções respiratórias agudas e gastrointestinais $(6,29)$ o que não foi evidenciado neste estudo, uma vez que o índice de internações foi de $11,1 \%$.

Dentre os distúrbios nutricionais identi- ficados entre as crianças menores de 2 anos, ressalta-se a presença do sobrepeso ou da obesidade (13\%), o que confirma a tendência nacional da transição nutricional caracterizada pelo aumento da obesidade infantil.

No Brasil, entre o período de 1989 a 2006, a prevalência de crianças com obesidade aumentou de $4,1 \%$ para $16 \%$ entre sexo masculino e de $2,4 \%$ para $11,8 \%$ entre o sexo feminino (5). Em estudo realizado em uma creche do município de São Paulo observouse que $17 \%$ das crianças menores de 5 anos apresentavam sobrepeso e $24 \%$ obesidade (30). Nesta mesma perspectiva, a avaliação antropométrica realizada em uma creche de Duque de Caxias, Rio de Janeiro, mostrou que um percentual de $21 \%$ de crianças com sobrepeso e 9\% com obesidade (31). Assim, verifica-se que a partir das últimas décadas do século 20, o excesso de peso constitui importante problema de saúde pública, podendo ser decorrente das mudanças nos hábitos do cotidiano, como: falta de aleitamento materno, modificações na alimentação, tendência ao sedentarismo, fatores econômicos, culturais e demográficos, entre outros.

A partir deste estudo realizado foi possível constatar a baixa prevalência de aleitamento materno entre as crianças que freqüentam uma creche comunitária. Além da prevalência de AME e AM, a investigação possibilitou caracterizar o perfil das mães que necessitam deixar seus filhos em uma creche, as condições de saúde e nutrição das crianças e verificar que as questões identificadas não mostraram associação com a duração do AME.

Considera-se oportuno referir que uma limitação do presente estudo pode ter ocorrido devido ao fato dos resultados obtidos expressarem a realidade específica de um número limitado de crianças menores de dois anos que freqüentam uma creche comunitária. Assim, destaca-se a necessidade do desenvolvimento de outras pesquisas mais abrangentes que permitam a generalização dos resultados e o preenchimento da lacuna 
existente na produção do conhecimento sobre a temática da amamentação de crianças que freqüentam creches.

Neste contexto, ressalta-se a importância da atuação de profissionais de saúde, em especial dos profissionais das equipes da Estratégia Saúde da Família, no ambiente das comunidades e creches, onde estes têm a possibilidade de realizar ações voltadas para o fortalecimento do vínculo da população com o serviço de saúde, auxiliar as mães no seguimento das orientações recebidas quanto aos cuidados com a alimentação infantil nos primeiros meses de vida e consequentemente de contribuir com a redução da morbi-mortalidade infantil.

Desta forma, conclui-se que as prevalências de AME e de AM apresentaram-se abaixo do esperado pela Organização Mundial de Saúde e que o conhecimento dos fatores associados à amamentação pode constituir em um importante subsídio para a implementação de ações voltadas para a promoção, proteção e apoio ao aleitamento materno.

\section{REFERÊNCIAS}

1. World Health Organization. Fifty-fourth World Health Assembly. Resolution WHA 54.2- Infant and young child nutrition. Geneva: World Health Organization; 2001.

2. Ministério da Saúde (Brasil). Saúde da Criança: Nutrição Infantil, Aleitamento Materno e Alimentação Complementar. Caderno de Atenção Básica, no 23 [Internet]. Brasília: Ministério da Saúde; 2009 [citado 28 marzo 2013]. 113 p. Disponível: http://www.sbp.com.br/src/uploads/2012/12/am_e_acl.pdf

3. Souza E, Minardi R, Eloiza S. Mitos e crenças sobre o aleitamento materno. Cien Saude Colet. 2011; 16(5): 24612468.
4. Brasil, Ministério da Saúde. Secretaria de Atenção à Saúde. Área Técnica de Saúde da Criança e Aleitamento Materno. Rede Amamenta Brasil: os primeiros passos (2007-2010) Brasília: Ministério da Saúde; 2011.

5. Brasil. Ministério da Saúde. Secretaria de atenção à saúde. Política nacional de alimentação e nutrição. Brasília: Ministério da Saúde; 2012.

6. World Health Organization. Indicators for assessing infant and young child feeding practices [Internet]. Washington: World Health Organization; 2007 [citado 28 marzo 2013]. 24 p. Disponível em: http://www.unicef.org/nutrition/files/ IYCF_updated_indicators_2008_part_1_ definitions.pdf

7. Dos Santos J, Spanó A, Azevedo F. O aleitamento materno enquanto uma prática construída: Reflexões acerca da evolução histórica da amamentação e desmame precoce no Brasil. Invest Educ Enferm. 2011; 29(2):315-321.

8. Barros A, Santos C, Menezes R. Concepções sobre maternidade, parto e amamentação em grupo de gestantes. Arq Bras Psicol. 2012; 64(1):139-155.

9. Ministério da Saúde (Brasil). II Pesquisa de Prevalência de Aleitamento Materno nas Capitais Brasileiras e Distrito Federal. Série C. Projetos, Programas e Relatórios [Internet]. Brasília: Ministério da Saúde; 2009 [citado 28 marzo 2013]. 108 p. Disponível em: http://www.sbp.com.br/src/ uploads/2012/12/pesquisa.pdf

10. Araújo A, Pires T. Identificando necessidades de crianças de creche e suas famílias: o uso do histórico de saúde como instrumento para um cuidado integral de pré-escolares. O Mundo da Saúde. 2009; 33(2):239-245.

11. Pinheiro N, Andrade M, Fujimori E. Amamentação em creches no Brasil. Rev. bras. cres. des. hum. 2009; 19(3):465-474. 12. Nogueira D, Ferreira D, Da Silva M, Fer- 
nandes P. Crianças em creche: acompanhamento do crescimento e desenvolvimento. R. Enferm. Cent. O. Min. 2012; 2(1):43-50.

13. Do Nascimento M, Silva G, Ferreira A, Domingues V, Gack L. Avaliação do estado nutricional e da saúde de crianças e adolescentes na prática assistencial do enfermeiro. Cogitare Enferm. 2013; 18(1):29-35.

14. World Health Organization. Anthro for personal computers: software for assessing growth and development of the world's children, version 3.2.2 [Internet]. Ginebra: WHO; 2011. [citado 02 nov 2013]. Disponível em: http://www.who. int/childgrowth/software/en/.

15. Siga A, Neves M, Zacca C. Prevalence of exclusive breastfeeding until the age of six months and maternal characteristics associated, in coverage area of a family health unit in the municipality of Pelotas, state of Rio Grande do Sul, Brazil, 2010. Epidemiol. Serv. Saúde. 2012; 21(3):431438.

16. Liaschi A, Oliveira M, Grubisch M, Hegeto $\mathrm{S}$, Bastos T. The prevalence and factors associated with exclusive breastfeeding in babies younger than six months in the city of Rolândia - PR. Nursing journal of minas gerais. 2013; 17(2):390-397.

17. Warkentin S, Viana KJ, Zapana PM, Taddei JAAC. Fatores associados à interrupção do aleitamento materno exclusivo antes dos seis meses em crianças matriculadas em creches públicas e filantrópicas do Município de São Paulo, Brasil. Nutrire: rev. Soc. Bras. Alim. Nutr. 2012; 37(2):105-117.

18. Vieira G, Martins C, Vieira T, Oliveira N, Silva L. Factors predicting early discontinuation of exclusive breastfeeding in the first month of life. J Pediatr. 2010; 86(5):441-4.

19. Instituto Brasileiro de Geografia e Estatística (IBGE). Anuário estatístico do
Brasil [Internet]. Rio de Janeiro: IBGE; 2012 [citado 02 nov 2013]. Disponível em: http://biblioteca.ibge.gov.br/visualizacao/periodicos/20/aeb_2012.pdf

20. Do Nascimento M, de Oliveira I, Romijn F. A Utilização do referencial metodológico de rede social na assistência de enfermagem a mulheres que amamentam. Rev Lat Am Enfermagem. 2009; 17(3):354360.

21. Souza E, Mitre R, Vieira M, Castro S, Amaral R, Lelis L. Rede social: desvendando a teia de relações interpessoais da nutriz. Physis. 2010; 20(1):261-8.

22. Couto M, De Oliveira I, Moreira E, Bastos L. Avaliação do apoio recebido para amamentar: significados de mulheres usuárias de Unidades Básicas de Saúde do estado do Rio de Janeiro. Ciênc saúde coletiva. 2010; 15(2):599-608.

23. Fundo das Nações Unidas para a Infância (UNICEF). Situação mundial da infância 2011. Adolescência: uma fase de oportunidades. Caderno Brasil [Internet]. 2011[citado 16 dez 2013]. 13 p. Disponível em: http://www.unicef.org/brazil/pt/ br_cadernoBR_SOWCR11(3).pdf.

24. Fein S. Aleitamento materno exclusivo para crianças menores de 6 meses. J Pediatr. 2009; 85(3):462-464.

25. Siqueira C, De Carvalho M, Couto M, Godoi A. Fatores associados à amamentação na primeira hora de vida. Rev. Saúde Pública. 2011; 45(1):69-78.

26. Parizoto G, Parada C, Venâncio S, Carvalhaes M. Tendência e determinantes do aleitamento materno exclusivo em crianças menores de 6 meses. J. Pediatr. 2009; 85(3):201-8.

27. Caminha M, Filho M, Serva V, Arruda I, Figueiroa J, Lira PI. Time trends and factors associated with breastfeeding in the state of Pernambuco, Northeastern Brazil. Rev Saude Publica. 2010; 44(2):240-8.

28. Brasil. Ministério da Saúde. Pesquisa nacional de demografia e saúde da criança e 
da mulher - PNSDS 2006: dimensões do processo reprodutivo e da saúde da criança. Brasília: Ministério da Saúde; 2008.

29. Siqueira C, Lazaro M, Couto M, Mello P. O papel do aleitamento materno na redução das hospitalizações por pneumonia em crianças brasileiras menores de 1 ano. J Pediatr. 2011; 87(5):399-404.
30. Morete M, Campos A. Ocorrência da obesidade infantil em pré-escolares de uma creche de São Paulo. Einstein. 2009; 7(2):170-75.

31. Dos Santos A, Leão L. Perfil antropométrico de pré-escolares de uma creche em Duque de Caxias, Rio de Janeiro. Rev. paul. pediatr. 2008; 26(3):218-24. 